\title{
Universal ventricular assist device for right and left circulatory support: the Cleveland Clinic concept
}

\author{
Jamshid H. Karimov, Anthony R. Polakowski, Kiyotaka Fukamachi, Takuma Miyamoto, Christine Flick \\ Department of Biomedical Engineering, Lerner Research Institute, Cleveland Clinic, Cleveland Clinic Lerner College of Medicine of Case Western \\ Reserve University, Cleveland, OH, USA \\ Correspondence to: Jamshid H. Karimov, MD, PhD. Cleveland Clinic, 9500 Euclid Avenue, Cleveland, OH 44195, USA. Email: karimoj@ccf.org.
}

Submitted Oct 07, 2020. Accepted for publication Nov 18, 2020.

doi: $10.21037 /$ acs-2020-cfmcs-22

View this article at: http://dx.doi.org/10.21037/acs-2020-cfmcs-22

With the observed evolution of left ventricular assist devices (LVADs) and the shift in patient phenotype, it is clear that continuous-flow (CF) LVADs are being increasingly utilized in those less ill (1). Despite incremental improvements in survival, a high frequency of complications remains an impediment to the wider adoption of CF pumps and more disruptive efforts are needed for truly effective neutralization of major adverse events (2). The need to address and augment pulsatility in circulatory support devices and the need for a clinical right ventricular assist device (RVAD) represent areas of current research and development. In addition, the auto-shut-off feature of the proposed device allows a unique fail-safe mode in the event of power interruption to help mitigate patient hazard. The requirement for the use of more than one device (biventricular assist device; BVAD) that requires a dedicated device controller for each pump can be addressed by designing a single driver unit that could automatically synchronize outputs for both devices, running both mechanical circulatory support (MCS) devices in concert (3) and further reduce the chances of controller failure.

The achievement of effective cardiac recovery is the main criteria for remission during LVAD support. Echocardiography remains a leading approach in examining ventricular ejection fraction, geometry and wall motion, though no consensus exists to address ventricular recovery (4). In the US, only approximately $5 \%$ of pump implants result in explant for recovery, based on current weaning protocols limited to algorithms for gradual pump speed reduction, verification of ventricular loading and observing aortic valve opening. Quantification of the competing contributions $(5,6)$ of regurgitant graft flow and forward pump flow, as well as the overall sum of these competing flows at low pump speeds, is an important aspect of deciding when a pump can be safely removed (4).

Although the detection of large regurgitant flow may serve as a marker for adequate left ventricular (LV) recovery, it also serves as a significant barrier for true assessment of $\mathrm{LV}$ function. LVAD forward flow contributes significantly to output, therefore, an accurate prediction of non-LVADsupported cardiac performance becomes more challenging as a recovering heart moves along the spectrum from zero to greater net flow (4). The advanced VAD design (AVAD) proposed by Cleveland Clinic was made to render the device-therapy suitable for both an LVAD and RVAD without modification, because at lower RVAD speeds, the aperture opening automatically decreases due to axial magnetic attraction which, in turn, attenuates hydraulic output for the RVAD application (7). This broad range of operation modes allows the device to operate as either an LVAD at high speed ( 3,300 rpm) or an RVAD at low speed $(\sim 2,300 \mathrm{rpm})$ without modification and with the same electronic hardware and software platform.

During operation, the axial position modulates along with cardiac cycle, so that the aperture opens with a surge in output at the start of systole and closes, attenuating the output, during diastole. In this way, the dynamic coupling of pump capacity is automatically and precisely timed to the action of the ventricle. In effect, the pump "pops its clutch" with every heartbeat, allowing a near physiologic aortic pulse pressure and flow pulse of approximately $10 \mathrm{~L} / \mathrm{min}$ (200\% flow pulsatility) when used as an LVAD. Because 
the rotor's axial movement is immediately sensed by the pump's hemodynamic environment, the parameters, such as heart rate, timing and relative strength of ventricular contractions, can be specified by the combination of speed, power and future rotor position signals.

The volute section of AVAD is axially offset from the impeller (8) to even out the pressures around the impeller, providing an axisymmetric pressure and flow distribution. This allows the device to operate over a wide range of flow/ speeds without driving adverse secondary flow patterns. In the event of incipient suction, the inlet cannula pressure will suddenly drop, and the aperture will decrease its opening, thereby reducing the output of the pump and forestalling the onset of suction until the controller can sense the event and lower the speed. Using the pulse amplification characteristic of the AVAD, speed can be adjusted either manually or by a speed control algorithm to maximize flow pulsatility. If the AVAD is operating at too high a speed, the system becomes depulsed. If the AVAD is too slow, the weak native pulse will not be amplified. Flow pulses can be recognized by the controller using current and rotor position signals. This allows real-time pulsatility feedback and the implementation of an automatic speed control by seeking the pump speed that maximizes pulsatility.

The computational flow dynamic analysis is currently pending and is expected to broaden our understanding of the pump's hemodynamics-whether employed as RVAD or LVAD support-and will hopefully provide insight into experimental results and guide the next steps in biocompatibility development. Additionally, the biocompatibility studies will be shedding more light on hemolysis parameters of AVAD. Our early simulation and feasibility studies have demonstrated promising results of the MCS device platform, universally applicable for left and right ventricular support $(7,9,10)$. The system is capable of delivering full systemic and pulmonary circulatory support over a wide range of hemodynamic conditions. Research and development is ongoing on simultaneous RVAD and LVAD application using the same device (dual device setup) for biventricular heart failure support. Ultimately, this will provide more insights on the best suitable platform and device configurations for biventricular circulatory support.

\section{Acknowledgments}

The technology is owned by Cleveland Clinic. The advanced ventricular assist device (AVAD) program is supported in part by Cleveland Clinic and through federal funding from the National Heart, Lung and Blood Institute (R21HL133871).

\section{Footnote}

Conflicts of Interest: The authors have no conflicts of interest to declare.

Open Access Statement: This is an Open Access article distributed in accordance with the Creative Commons Attribution-NonCommercial-NoDerivs 4.0 International License (CC BY-NC-ND 4.0), which permits the noncommercial replication and distribution of the article with the strict proviso that no changes or edits are made and the original work is properly cited (including links to both the formal publication through the relevant DOI and the license). See: https://creativecommons.org/licenses/by-nc-nd/4.0/.

\section{References}

1. Kormos RL, Cowger J, Pagani FD, et al. The Society of Thoracic Surgeons Intermacs Database Annual Report: Evolving Indications, Outcomes, and Scientific Partnerships. Ann Thorac Surg 2019;107:341-53.

2. Kirklin JK, Pagani FD, Kormos RL, et al. Eighth annual INTERMACS report: Special focus on framing the impact of adverse events. J Heart Lung Transplant 2017;36:1080-6.

3. Medvedev AL, Karimov JH, Kuban BD, et al. Unlocking the box: basic requirements for an ideal ventricular assist device controller. Expert Rev Med Devices 2017;14:393-400.

4. Kirklin JK. Mock loop revelations and the calculus for recovery. J Thorac Cardiovasc Surg 2015;150:348-9.

5. Sunagawa G, Byram N, Karimov JH, et al. The Contribution to Hemodynamics Even at Very Low Pump Speeds in the HVAD. Ann Thorac Surg 2016;101:2260-4.

6. Sunagawa G, Byram N, Karimov JH, et al. In vitro hemodynamic characterization of HeartMate II at 6000 rpm: Implications for weaning and recovery. J Thorac Cardiovasc Surg 2015;150:343-8.

7. Miyamoto T, Kado Y, Horvath DJ, Kuban BD, Sale S, Fukamachi K, et al. An advanced universal circulatory assist device for left and right ventricular support: First report of an acute in vivo implant. JTCVS Open 2020;3:140-8.

8. Womersley JR. Method for the calculation of velocity, 
rate of flow and viscous drag in arteries when the pressure gradient is known. J Physiol 1955;127:553-63.

9. Karimov JH, Horvath D, Kado Y, et al. Mechanical Circulatory Support for Biventricular Heart Failure Using Advanced Ventricular Assist Device. J Heart Lung

Cite this article as: Karimov JH, Polakowski AR, Fukamachi K, Miyamoto T, Flick C. Universal ventricular assist device for right and left circulatory support: the Cleveland Clinic concept. Ann Cardiothorac Surg 2021;10(2):271-273. doi:10.21037/acs2020-cfmcs-22
Transplant 2020;39:S414.

10. Miyamoto T, Byram N, Karimov JH, et al. The design modification of advanced ventricular assist device to enhance pulse augmentation and regurgitant flow shut-off. Artif Organs 2019;43:961-5. 